\title{
Integrating experimental and computational approaches for the proteogenomic characterizations of microbial communities
}

\author{
Robert L Hettich ${ }^{1 *}$, Alison L Russell ${ }^{1}$, Nathan C VerBerkmoes ${ }^{1}$, Manesh Shah ${ }^{1}$, Claire Fraser-Liggett ${ }^{2}$, \\ Janet $\mathrm{K}$ Jansson ${ }^{3}$
}

From UT-ORNL-KBRIN Bioinformatics Summit 2010

Cadiz, KY, USA. 19-21 March 2010

\section{Background}

The human gastrointestinal tract is a complex ecosystem containing a delicate balance of human and microbial cells involved in an intricate symbiotic relationship. In general, the microbial constituency helps maintain a healthy environment and aids in the efficient digestion. However, environmental and/or genetic factors may result in an altered bacterial composition that manifests in a diseased condition, such as Crohn's disease. The recent availability of genomic-based molecular technologies such as whole community genome sequencing and whole community proteomics have provided unique capabilities of profiling the compositions and activities of this microbiome without having to cultivate its membership.

\section{Materials and methods}

We are utilizing a non-targeted, mass spectrometrybased proteomics approach to identify the microbial proteins in fecal samples from human twins. Proteome samples were analyzed with technical duplicates via a multidimensional LC tandem mass spectrometric approach on a hybrid linear ion trap-Orbitrap. The proteome measurements identified $>2000$ proteins for each sample and replicate.

\section{Results and conclusion}

Amongst the microbial genomes, Bacteroides thetaiotaomicron,Bifidobacterium longum, Bacteroides fragilis and Bifidobacterium adolescentis were the most highly identified species, as expected since these are known to be among the most abundant bacteria in the human gut. Interestingly, the majority of the microbial proteins that were identified were classified into COG categories for translation, energy generation, and carbohydrate metabolism. The latter category is especially interesting in that it reveals one of the synergistic relationships between humans and microbes in this ecosystem. Surprisingly, a number of innate human immunity proteins were also observed, suggesting a level of human regulation of microbial abundance. A number of abundant unknown proteins were also identified. The results of this study demonstrate that it is possible to obtain high quality, extensive protein identifications from translated metagenome sequence data collected from distinct human individuals.

\section{Acknowledgements}

Research support provided by NIH-HMP Demonstration Program. Oak Ridge National Laboratory is managed by UT-Battelle, LLC, for the U.S. Department of Energy under Contract No. DE-AC05-000R22725.

\section{Author details}

'Oak Ridge National Laboratory, Oak Ridge, TN 37831, USA. ²University of Maryland School of Medicine, Baltimore, MD 21201, USA. 'awrence Berkeley National Laboratory, Berkeley, CA 94720, USA.

Published: 23 July 2010

doi:10.1186/1471-2105-11-S4-O6

Cite this article as: Hettich et al:: Integrating experimental and computational approaches for the proteogenomic characterizations of microbial communities. BMC Bioinformatics 2010 11(Suppl 4):06.

* Correspondence: hettichrl@ornl.gov

'Oak Ridge National Laboratory, Oak Ridge, TN 37831, USA 